\section{Eficácia da associação de fipronil e permetrina no controle de Ctenocephalides felis felis e Rhipicephalus sanguineus sensu lato em cães artificialmente infestados}

\author{
Efficacy of the fipronil and permethrin association control of \\ Ctenocephalides felis felis e Rhipicephalus sanguineus sensu lato \\ in artificially infested dogs
}

\begin{abstract}
Monique Moraes Lambert', Diefrey Ribeiro Campos', Barbara Rauta de Avelar', Rosangela Rodrigues dos Santos', Debora Azevedo Borges ${ }^{2}$, Rayane Christine Pereira de Assis ${ }^{3}$, Fabio Barbour Scott ${ }^{4}$ \& Katherina Coumendouros ${ }^{4 *}$ 'Médicos veterinários, MSc. Programa de Pós-graduação em Ciências Veterinárias - PPGCV, Universidade Federal Rural do Rio de Janeiro - UFRRJ, Seropédica, RJ, Brasil

2Médica veterinária. Programa de Pós-graduação em Ciências Veterinárias - PPGCV, Universidade Federal Rural do Rio de Janeiro - UFRRJ, Seropédica, RJ, Brasil

${ }^{3}$ Médica veterinária, Residente. Programa de Residência Multiprofissional em Saúde, Universidade Federal Rural do Rio de Janeiro - UFRRJ, Seropédica, RJ, Brasil

${ }^{4}$ Médicos veterinários, DSc, Docentes. Departamento de Parasitologia Animal, Instituto de Veterinária, Universidade Federal Rural do Rio de Janeiro - UFRRJ, Seropédica, RJ, Brasil
\end{abstract}

\section{Resumo}

Este estudo foi realizado com o objetivo de avaliar a eficácia carrapaticida e pulicida de uma formulação "spot on" contendo a associação de fipronil 6,01\% + permetrina 44,88\%, no controle de Rhipicephalus sanguineus e Ctenocephalides felis felis em cães artificialmente infestados. Foram utilizados 12 cães da raça beagle, de ambos os sexos, com idade de dois a cinco anos, divididos em dois grupos: controle e tratado. Os cães foram infestados com 100 exemplares de $C$. felis felis e 50 exemplares de $R$. sanguineus. As infestações foram realizadas nos dias, $-2,+5,+12,+19,+26,+33$ para carrapato e com pulgas nos dias, $-2+5,+12,+19,+26,+33,+40,+47+54$. A avaliação da eficácia carrapaticida ocorreu nos dias $+2,+7,+14,+21,+28,+35$. A avaliação da eficácia pulicida seguiram nos dias $+2,+7,+14,+21,+28,+35,+42,+49,+56$ para pulgas. A eficácia carrapaticida foi de: 100\%; 96,91\%; 92,86\%; 91,10\%; 54,91\% e 51,95\% para os dias $+2,+7,+14,+21,+28$ e +35 respectivamente, enquanto a eficácia pulicida foi de $100 \%$ até o dia +28 e de $97,44 \%$ para do dia +35 , 97,41\% para o dia +42 , $82,87 \%$ para o dia +49 e 75,00\% para o dia +56 . Com base nos resultados obtidos neste estudo é possível concluir que a associação de fipronil 6,01\% e permetrina 44,88\% foi eficaz para o controle de C. felis felis e $R$. sanguineus em cães artificialmente infestados.

Palavras-chave: pulga, carrapato, controle.

\begin{abstract}
This study was carried out with the objective of evaluating the acaricide and insecticide efficacy of a formulation containing the association spot on of fipronil (6.01\%) and permethrin (44.88\%), in control of Rhipicephalus sanguineus sensu lato and Ctenocephalides felis felis in experimentally infested dogs. Were used in this study twelve beagle dogs were divided in two groups: control and treated. The dogs were infested with 50 pairs of $C$. felis felis and 25 pairs of $R$. sanguineus for evaluation of efficacy. Infestations were carried out on days $-2,+5,+12,+19,+26,+33$ for ticks and with fleas on days, $-2+5,+12,+19,+26,+33,+40,+47+54$. On the days, $+2,+7,+14,+21,+28,+35$ the evaluations for ticks were performed and on days, $+2,+7,+14,+21,+28,+35,+42,+49,+56$ for fleas. Acaricide efficacy was: 100\%; 96.91\%; 92.86\%; 91.10\%; 54.91\% and 51.95\% for days $+2,+7,+14,+21,+28$ and +35 respectively, while pulicidal efficacy was $100 \%$ up to day +28 and $97.44 \%$ for of day $+35,97.41 \%$ for day $+42,82.87 \%$ for day +49 and $75.00 \%$ for day +56 . Based on the results obtained in this study it is possible to conclude that the association of fipronil $6.01 \%$ and permethrin $44.88 \%$ was effective for the control of $C$. felis felis and $R$. sanguineus in artificially infested dogs.
\end{abstract}

Keywords: flea, tick, control.
BJ

p-ISSN 0100-2430

e-ISSN 2527-2179

○
Como citar: Lambert, M. M., Campos, D. R., Avelar, B. R., Santos, R. R., Borges, D. A., Assis, R. C. P., Scott, F. B., \& Coumendouros, K. (2017). Eficácia da associação de fipronil e permetrina no controle de Ctenocephalides felis felis e Rhipicephalus sanguineus sensu lato em cães artificialmente infestados. Brazilian Journal of Veterinary Medicine, 39(4), 246-251. doi: 10.29374/2527-2179.bjvm019517

Fonte de financiamento: Fundação de Apoio à Pesquisa Científica e Tecnológica da Universidade Federal Rural do Rio de Janeiro.

Conflito de interesses: Os autores declaram não haver conflito de interesses que precisam ser informados.

Recebido: Agosto 24, 2017.

Aceito: Dezembro 02, 2017

O estudo foi realizado na Universidade Federal Rural do Rio de Janeiro - UFRRJ, Seropédica, RJ, Brasil.

\section{*Correspondência}

Katherina Coumendouros

Departamento de Parasitologia Animal, Instituto de Veterinária, Universidade Federal Rural do Rio de Janeiro - UFRRJ

Rodovia BR 465, Km 07, s/n, Zona Rural, CEP 23890-000 - Seropédica (RJ), Brasil E-mail:katherinac@ufrrj.com

Copyright Lambert et al. Este é um artigo publicado em acesso aberto (Open Access) sob a licença Creative Commons Attribution Non-Commercial, que permite uso, distribuição e reprodução em qualquer meio, sem restrições desde que sem fins comerciais e que o trabalho original seja corretamente citado. 


\section{Introdução}

Pulgas e carrapatos são ectoparasitos que causam incomodo a animais de companhia e seus tutores. Além do desconforto, são responsáveis pela transmissão de diversos agentes patogênicos, causam irritação, anemia, e podem provocar o quadro de dermatite alérgica a presença de ectoparasitos (Dantas-Torres, 2008; Patterson, 2015).

Entre os patógenos transmitidos por carrapatos no Brasil podemos citar: Babesia spp., Rangelia vitalli, Ehrlichia canis, Anaplasma spp., Ricketisia spp. e Hepatozoon canis, Mycoplasma haemocanis (Parker et al., 1933; Sen, 1933; Regendanz \& Muniz, 1936; Groves et al., 1975; Nordgren \& Craig, 1984; Simpson et al., 1991; Coutinho et al., 2005; Loretti \& Barros, 2005).

As pulgas do gênero Ctenocephalides estão associadas a transmissão de patógienos como Ricktisia spp., Bartonella hanselae eMycoplasma spp. (Mexas et al., 2002; Gehrke et al., 2009; Hornok et al., 2010). São também considerados hospedeiros intermediários de helmintos como: Dipylidium caninum e Acanthocheilonema reconditum (Nelson, 1962; Pugh, 1987).

Os piretróides são grupo de ectoparasiticidas derivados sinteticamente a partir das flores de Chrysanthemum cinerariaefolium. Possuem ação inseticida e carrapaticida e apresentam baixa toxicidade para cães (Bowers, 1985). Seu mecanismo de ação é por modificar os canais de sódio não sendo seletivo somente para invertebrados (Schreck et al., 1978). Durante anos produtos com esses ativos foram utilizados no controle de pulgas e carrapatos em cães. Atualmente, devido a sua ação repelente (efeito "hot-foot") para ácaros e insetos seu uso tem sido empregado em associação com outros ativos principalmente com os neonicotinóides (Blagburn \& Dryden, 2009). Os principais piretroides utilizados em associação com outros grupamentos, para o controle de $C$. felis felis e $R$. sanguineus em cães, são flumetrina e permetrina (Hellmann et al., 2003; Dantas-Torres, 2008).

O fipronil possui excelente ação inseticida e acaricida. Seu principal mecanismo de ação é sobre o receptor de íons cloretos GABA. O GABA possui a função de permitir a entrada de íons cloreto no interior da célula neural, fazendo com que retorne ao seu potencial de repouso, mantendo o potencial elétrico da membrana. O bloqueio destes canais ocasionado pelo fipronil reduz a inibição neuronal e leva à hiper-excitação do sistema nervoso central, cursando com paralisia, convulsões e morte, entretanto esta molécula não possui ação de repelência e seu efeito "knock down" é baixo (Zhao et al., 2003; Zheng et al., 2003; Zhao et al., 2004).

A associação de um permetrina com o fipronil poderá promover uma potencialização dos efeitos para um melhor controle destes ectoparasitos de cães. Neste caso teríamos uma formulação com ação imediata ("knock down") e repelência determinados pela permetrina, associado com uma eficácia acaricida e inseticida prolongada determinada pelo fipronil.

O objetivo deste trabalho foi avaliar a eficácia de uma associação fipronil 6,01\% e permetrina 44,88\% na formulação "spot on" com no controle de C. felis felis e R. sanguineus em cães.

\section{Materiais e métodos}

Este estudo foi aprovado pelo Comitê de Ética de Uso de Animal (CEUA) da Fundação de Apoio à Pesquisa Científica e Tecnológica (FAPUR), da UFRRJ, aprovado no dia 07 de março de 2013.

Os animais foram alojados individualmente no dia -14 para aclimatação e no dia -7 estes foram infestados com 100 exemplares de $C$. felis felis ( 50 machos e 50 fêmeas) e 25 casais de R. sanguineus adultos, não alimentados e com idade aproximadamente de 14 dias para realização do ranqueamento. Após 48 horas (dia -5), todos os animais foram examinados para a retirada manual de todos os carrapatos e posteriormente penteados para retirada de todas as pulgas. Para a randomização do ensaio, foi efetuado um sorteio de cada animal, do mais parasitado para o menos parasitado, alocando-se um animal em cada grupo, e assim sucessivamente até que se completassem as 12 repetições distribuídas nos dois grupos.

Após o ranqueamento as infestações ocorreram conforme descrito acima nos dias $-2,+5,+12,+19,+26,+33$, para carrapato. Para pulga as infestações seguiram nos dias $+40,+47$ e +54 . A avaliação da eficácia ocorreram sempre após 48h das infestações, com exceção do dia-2 que foi realizada $72 \mathrm{~h}$, correspondendo aos dias $+2,+7,+14,+21,+28 \mathrm{e}+35$, para $R$. sanguineus. Para $C$. felisfelis as avaliações foram efetuadas nos dias $+2,+7,+14,+21,+28$ e $+35+42,+49$ e +56 . 
A formulação empregada foi adaptada da proposta por Marchiondo et al. (2013) para ensaios que tem como objetivo avaliar a eficácia de formulações para pulgas e carrapatos em cães e gatos.

No dia $\mathrm{O}$ (dia do tratamento) foi administrado nos cães do grupo tratados pela via "spot on" a associação de fipronil 6,01\% com permetrina 44,88\% em dose única. O conteúdo das pipetas foi aplicado diretamente sobre a pele, ao longo do pescoço no sentido contra o pelo do animal.

O cálculo das eficácias da formulação testada foi realizado baseado nas seguintes fórmulas:

Eficácia Pulicida (\%) = (número médio de pulgas vivas recuperadas no grupo controle número médio de pulgas vivas recuperadas no grupo tratado) / (número médio de pulgas vivas recuperadas no grupo controle) x 100.

Eficácia carrapaticida (\%) = (número médio de carrapatos vivos e fixados recuperados no grupo controle - número médio de carrapatos vivos e fixados recuperados no grupo medicado) / (número médio de carrapatos vivos e fixados recuperados no grupo controle) x 100.

Para análise estatística os dados foram testados quando a normalidade pelo teste de Shapiro-Wilks, Como não apresentaram distribuição normal, foram transformados em $\log _{10}(x+1)$ e submetidos à análise do Teste T. O nível de significância considerado foi de $95 \%$ ( $\mathrm{P} \leq 0,05)$. As análises estatísticas foram realizadas pelo programa estatístico computacional BioEstat 5.3 .

\section{Resultados}

A recuperação de pulgas e carrapatos, pré-tratamento, de todos os cães foi alta. Sendo superior a 20\% para $R$. sanguineus e maior que 25\% para C. felis felis. Esse percentual é definido por Marchiondo et al. (2013) como o mínimo aceitável em relação ao total de parasitos que foram utilizados na infestação que define o ranqueamento, de forma a conferir aos resultados do estudo resposta confiável sob o ponto de vista estatístico. Não foi observada diferença estatística $(\mathrm{p}>0,05)$ entre as médias do grupo controle e tratado na avaliação pré-tratamento (Tabela 1).

A média aritmética de pulgas vivas recuperadas no grupo controle foi de: 51,00 para o dia +2; 52,83 para o dia $+7 ; 50,67$ para o dia $+14 ; 46,67$ para o dia $+21 ; 54$ para o dia $+28 ; 58,5$ para o dia +35 ; 57,83 para o dia $+42 ; 60,33$ para o dia +49 e 61,33 para o dia +56 . Para o grupo tratado as médias foram: $0 ; 0 ; 0 ; 0 ; 0 ; 1,50 ; 1,50 ; 10,33 ; 15,33$, respectivamente para os dias $+2,+7,+14,+21,+28,+35$, $+42,+49$ e +56 . Foi observada diferença estatística $(\mathrm{p}<0,05)$ para as médias do grupo controle e tratado em todas as avaliações pós-tratamento. Os resultados de média, desvio padrão estão demonstrados na Tabela 1.

A eficácia pulicida da formulação testada foi de 100\% até o dia +28 e de 97,44\% para do dia $+35,97,41 \%$ para o dia $+42,82,87 \%$ para o dia +49 e $75,00 \%$ para o dia +56 .

Tabela 1. Média Aritmética da contagem de pulgas (Ctenocephalides felis felis) vivas nos cães do grupo controle e tratados e eficácia da formulação Fipronil 6,01\% mais Permetrina 44,8\%, em cães infestados artificialmente.

\begin{tabular}{|c|c|c|c|}
\hline \multirow{2}{*}{ Dia Experimental } & \multirow{2}{*}{$\begin{array}{c}\text { Grupo Controle } \\
\text { Média* }^{*}\end{array}$} & \multicolumn{2}{|c|}{ Grupo Tratado } \\
\hline & & Média* & Eficácia \\
\hline+2 & $51,00^{\mathrm{a}} \pm 7,90$ & $\mathrm{O}^{\mathrm{b}} \pm \mathrm{O}$ & $100 \%$ \\
\hline+7 & $52,83^{\mathrm{a}} \pm 5,42$ & $\mathrm{O}^{\mathrm{b}} \pm 0$ & $100 \%$ \\
\hline+14 & $50,67^{a} \pm 6,89$ & $\mathrm{O}^{\mathrm{b}} \pm \mathrm{O}$ & $100 \%$ \\
\hline+21 & $46,67^{\mathrm{a}} \pm 2,73$ & $\mathrm{O}^{\mathrm{b}} \pm \mathrm{O}$ & $100 \%$ \\
\hline+28 & $54,00^{\mathrm{a}} \pm 5,10$ & $\mathrm{O}^{\mathrm{b}} \pm \mathrm{O}$ & $100 \%$ \\
\hline+35 & $58,50^{\mathrm{a}} \pm 2,35$ & $1,50^{\mathrm{b}} \pm 1,76$ & $97,44 \%$ \\
\hline+42 & $57,83^{\mathrm{a}} \pm 6,24$ & $1,50^{\mathrm{b}} \pm 0,55$ & $97,41 \%$ \\
\hline+49 & $60,33^{\mathrm{a}} \pm 4,97$ & $10,33^{b} \pm 3,67$ & $82,87 \%$ \\
\hline+56 & $61,33^{\mathrm{a}} \pm 9,58$ & $15,33^{b} \pm 5,24$ & $75,00 \%$ \\
\hline
\end{tabular}

*Média Geométrica; Letras iguais dentro da mesma coluna, $p$-value>0,05 (não há diferença estatística); Letras diferentes entre as colunas, p-value $<0,05$ (há diferença estatística). 
Tabela 2. Média Aritmética da contagem de carrapatos (Rhipicephalus sanguineus) vivos e fixados nos cães do grupo controle e tratados e eficácia da associação de Fipronil 6,01\% mais Permetrina 44,8\%, em cães infestados artificialmente.

\begin{tabular}{|c|c|c|c|}
\hline \multirow{2}{*}{ Dia Experimental } & \multirow{2}{*}{$\begin{array}{c}\text { Grupo Controle } \\
\text { Média* }\end{array}$} & \multicolumn{2}{|c|}{ Grupo Tratado } \\
\hline & & Média* & Eficácia \\
\hline+2 & $21,33^{\mathrm{a}} \pm 5,72$ & $\mathrm{O}^{\mathrm{b}} \pm \mathrm{O}$ & $100 \%$ \\
\hline+7 & $27,00^{\mathrm{a}} \pm 5,10$ & $0,83^{b} \pm 0,98$ & $96,91 \%$ \\
\hline+14 & $30,33^{a} \pm 8,71$ & $2,17^{b} \pm 1,72$ & $92,86 \%$ \\
\hline+21 & $39,33^{\mathrm{a}} \pm 6,89$ & $3,50^{\mathrm{b}} \pm 1,87$ & $91,10 \%$ \\
\hline+28 & $37,33^{\mathrm{a}} \pm 6,15$ & $16,83^{\mathrm{b}} \pm 9,43$ & $54,91 \%$ \\
\hline+35 & $42,67^{\mathrm{a}} \pm 6,86$ & $20,50^{b} \pm 7,45$ & $51,95 \%$ \\
\hline
\end{tabular}

*Média Geométrica; Letras iguais dentro da mesma coluna, p-value>0,05 (não há diferença estatística); Letras diferentes entre as colunas, p-value < 0,05 (há diferença estatística).

A recuperação de carrapatos vivos e fixados nos cães do grupo controle apresentou média de: 21,33 para o dia $+2 ; 27,00$ para o dia $+7 ; 30,33$ para o dia $+14 ; 39,33$ para o dia $+21 ; 37,33$ para o dia $+28 ; 42,67$ para o dia +35. Para o grupo tratado as médias foram: 0; 0,83;2,17;3,5;16,83 e20,5, respectivamente para os dias $+2,+7,+14,+21,+28,+35$. Foi observada diferença estatística $(\mathrm{p}<0,05)$ para as médias do grupo controle e tratado em todas as avaliações pós-tratamento. Os resultados de média, desvio padrão estão demonstrados na Tabela 2.

A eficácia carrapaticida observada foi de: 100\%; 96,91\%; 92,86\%; 91,10\%; 54,91\% e 51,95\%, respectivamente para os dias $+2,+7,+14,+21,+28$ e +35 .

\section{Discussão}

De acordo com Marchiondo et al. (2013) um bom ectoparasiticida deve ter eficácia pulicida e carrapaticida superior a 90\%. Sendo assim, com base nos resultados obtidos neste estudo a associação de fipronil-permetrina demonstrou excelente eficácia no controle de $R$. sanguineus por 21 dias e para C. felis felis 42 dias.

Esta associação demonstrou eficácia semelhante a outros estudos realizados para os carrapatos R. sanguineus, Ixodes ricinus e pulgas C. felis felis (Otranto et al., 2005; Bonneau et al., 2015; Beugnet et al., 2016; Halos et al., 2016).

O fipronil possui excelente ação inseticida e carrapaticida. Contudo, especialmente para carrapatos, age de forma progressiva e só elimina estes parasitos a partir de 24 a 48 horas após a infestação. Desta foram o baixo efeito "knock down" do fipronil quando isoladamente empregado, não promove ação capaz de impedir que pulgas e carrapatos se alimentem até que seus efeitos atinjam os mais elvados níveis, consequentemente não seria capaz de previner o desenvolvimento de quadros de DAPE e a transmissão de agentes patogênicos para cães (Cruthers et al., 2001; Brianti et al., 2010).

Apesar da permetrina possuir ação inseticida e acaricida, sua persistência não é acentuada. Sua associação ao fipronil seria desejável pois acrecentaria ação repelente e efeito "knock-down", minimizando a possibilidade que que carrapatos e pulgas consigam se alimentar e prevenindo, desta forma, a transmissão de hemoparasitos como Babesia spp., Ehrlichia canis (Jongejan et al., 2015; Navarro et al., 2015).

A eficácia da repelência desta associação não foi avaliada neste estudo. No entanto, estudos realizados utilizando produtos com fipronil epermetrina mostraram sua eficácia na repelência de pulgas e carrapatos, mostrando desta forma um efeito associativo entre as duas moléculas (Endris et al., 2000; Beugnet et al., 2015).

Com base nos resultados obtidos neste estudo é possível concluir que a associação de fipronil 6,01\% e permetrina 44,88\% foi eficaz para o controle de $C$. felis felis e $R$. sanguineus em cães artificialmente infestados. 


\section{Referências}

Beugnet, F., Halos, L., Liebenberg, J., \& Fourie, J. (2016). Assessment of the prophylactic speed of kill of Frontline Tri-Act ${ }^{\circledR}$ against ticks (Ixodes ricinus and Rhipicephalus sanguineus) on dogs. Parasite, 23, 2-6. http://dx.doi. org/10.1051/parasite/2016002. PMid:26795064.

Beugnet, F., Soll, M., Bouhsira, E., \& Franc, M. (2015). Sustained speed of kill and repellency of a novel combination of fipronil and permethrin against Ctenocephalides canis flea infestations in dogs. Parasites \& Vectors, 8(1), 52. http://dx.doi.org/10.1186/s13071-015-0680-1. PMid:25622656.

Blagburn, B. L., \& Dryden, M. W. (2009). Biology, treatment, and control of flea and tick infestations. Veterinary Clinics: Small Animal Practice, 39(6), 1173-1200. PMid:19932369.

Bonneau, S., Reymond, N., Gupta, S., \& Navarro, C. (2015). Efficacy of a fixed combination of permethrin 54.5\% and fipronil 6.1\%(Effitix $\left.{ }^{\circledR}\right)$ in dogs experimentally infested with Ixodes ricinus. Parasites \& Vectors, 8(1), 204. http://dx.doi.org/10.1186/s13071-015-0805-6. PMid:25884999.

Bowers, W. S. (1985). Phytochemical resources for plant protection. Recent Advances in the Chemistry of Insect Control, 1, 272-292.

Brianti, E., Pennisi, M. G., Brucato, G., Risitano, A. L., Gaglio, G., Lombardo, G., Malara, D., Fogliazza, A., \& Giannetto, S. (2010). Efficacy of the fipronil 10\%+(S)-methoprene 9\% combination against Rhipicephalus sanguineus in naturally infested dogs: speed of kill, persistent efficacy on immature and adult stages and effect of water. Veterinary Parasitology, 170(1-2), 96-103. http://dx.doi.org/10.1016/j.vetpar.2010.01.033. PMid:20185241.

Coutinho, M. T. Z., Bueno, L. L., Sterzik, A., Fujiwara, R. T., Botelho, J. R., Maria, M., Genaro, O., \& Linardi, P. M. (2005). Participation of Rhipicephalus sanguineus (Acari: Ixodidae) in the epidemiology of canine visceral leishmaniasis. Veterinary Parasitology, 128(1-2), 149-155. http://dx.doi.org/10.1016/i.vetpar.2004.11.011. PMid:15725545.

Cruthers, L., Slone, R. L., Guerrero, A. J., \& Robertson-Plouch, C. (2001). Evaluation of the speed of kill of fleas and ticks with Frontline Top Spot in dogs. Veterinary Therapeutics, 2(2), 170-174. PMid:19753710.

Dantas-Torres, F. (2008). The brown dog tick, Rhipicephalus sanguineus (Latreille, 1806) (Acari: Ixodidae): from taxonomy to control. Veterinary Parasitology, 152(3-4), 173-185. http://dx.doi.org/10.1016/j.vetpar.2007.12.030. PMid:18280045.

Endris, R. G., Matthewson, M. D., Cooke, M. D., \& Amodie, D. (2000). Repellency and efficacy of 65\% permethrin and 9.7\% fipronil against Ixodes ricinus. Veterinary Therapeutics: Research in Applied Veterinary Medicine, 1(3), 159-168. PMid:19757578.

Gehrke, F. S., Gazeta, G. S., Souza, E. R., Ribeiro, A., Martelli, M. T., \& Schumaker, T. T. S. (2009). Rickettsia rickettsii, Rickettsia felis and Rickettsia sp. TwKM03 infecting Rhipicephalus sanguineus and Ctenocephalides felis collected from dogs in a Brazilian spotted fever focus in the State of Rio De Janeiro/Brazil. Clinical Microbiology and Infection, 15(Suppl 2), 267-268. http://dx.doi.org/10.1111/j.1469-0691.2008.02229.x. PMid:19298400.

Groves, M. G., Dennis, G. L., Amyx, H. L., \& Huxsoll, D. L. (1975). Transmission of Ehrlichia canis to dogs by ticks (Rhipicephalus sanguineus). American Journal of Veterinary Research, 36(7), 937-940. PMid:1147359.

Halos, L., Fourie, J. J., Fankhauser, B., \& Beugnet, F. (2016). Knock-down and speed of kill of a combination of fipronil and permethrin for the prevention of Ctenocephalides felis flea infestation in dogs. Parasites \& Vectors, 9(1), 57. http://dx.doi.org/10.1186/s13071-016-1345-4. PMid:26830931.

Hellmann, K., Knoppe, T., Krieger, K., \& Stanneck, D. (2003). European Multicenter Field Trial on the Efficacy and Safety of a topical Formulation of Imidacloprid and Permethrin (AdvantixTM) in Dogs naturally infested with Ticks and/or Fleas. Parasitology Research, 90(0), 125-126. http://dx.doi.org/10.1007/s00436-003-0912-8.

Hornok, S., Meli, M. L., Perreten, A., Farkas, R., Willi, B., Beugnet, F., Lutz, H., \& Hofmann-Lehmann, R. (2010). Molecular investigation of hard ticks (Acari: Ixodidae) and fleas (Siphonaptera: Pulicidae) as potential vectors of rickettsial and mycoplasmal agents. Veterinary Microbiology, 140(1-2), 98-104. http://dx.doi.org/10.1016/j. vetmic.2009.07.013. PMid:19660880.

Jongejan, F., De Vos, C., Fourie, J. J., \& Beugnet, F. (2015). A novel combination of fipronil and permethrin (Frontline Tri-Act ${ }^{\circledast} /$ Frontect $^{\circledast}$ ) reduces risk of transmission of Babesia canis by Dermacentor reticulatus and of Ehrlichia canis by Rhipicephalus sanguineus ticks to dogs. Parasites \& Vectors, 8(1), 602. http://dx.doi.org/10.1186/s13071015-1207-5. PMid:26586365.

Loretti, A. P., \& Barros, S. S. (2005). Hemorrhagic disease in dogs infected with an unclassified intraendothelial piroplasm in southern Brazil. Veterinary Parasitology, 134(3-4), 193-213. http://dx.doi.org/10.1016/j.vetpar.2005.07.011. PMid:16153781.

Marchiondo, A. A., Holdsworth, P. A., Fourie, L. J., Rugg, D., Hellmann, K., Snyder, D. E., \& Dryden, M. W. (2013). World Association for the Advancement of Veterinary Parasitology (WAAVP): guidelines for evaluating the efficacy of parasiticides for the treatment, prevention and control of flea and tick infestations on dogs and cats. Veterinary Parasitology, 194(1), 84-97. http://dx.doi.org/10.1016/j.vetpar.2013.02.003. PMid:23741753.

Mexas, A. M., Hancock, S. I., \& Breitschwerdt, E. B. (2002). Bartonella henselae and Bartonella elizabethae as potential canine pathogens. Journal of Clinical Microbiology, 40(12), 4670-4674. http://dx.doi.org/10.1128/ JCM.40.12.4670-4674.2002. PMid:12454170. 
Navarro, C., Reymond, N., Fourie, J., Hellmann, K., \& Bonneau, S. (2015). Prevention of Babesia canis in dogs: efficacy of a fixed combination of permethrin and fipronil (Effitix $\left.{ }^{\circledR}\right)$ using an experimental transmission blocking model with infected Dermacentor reticulatus ticks. Parasites \& Vectors, 8(1), 32. http://dx.doi.org/10.1186/ s13071-015-0645-4. PMid:25595325.

Nelson, G. S. (1962). Dipetalonema reconditum (Grassi, 1889) from the dog with a note on its development in the flea, Ctenocephalides felis and the louse, Heterodoxus spiniger. Journal of Helminthology, 36(03), 297-308. http://dx.doi.org/10.1017/S0022149X00023968.

Nordgren, R. M., \& Craig, T. M. (1984). Experimental transmission of the Texas strain of Hepatozoon canis. Veterinary Parasitology, 16(3-4), 207-214. http://dx.doi.org/10.1016/0304-4017(84)90038-4. PMid:6542719.

Otranto, D., Lia, R. P., Cantacessi, C., Galli, G., Paradies, P., Mallia, E., \& Capelli, G. (2005). Efficacy of a combination of imidacloprid 10\%/permethrin 50\% versus fipronil 10\%/(S)-methoprene $12 \%$, against ticks in naturally infected dogs. Veterinary Parasitology,130(3-4), 293-304. http://dx.doi.org/10.1016/j.vetpar.2005.04.014. PMid:15893431.

Parker, R. R., Philip, C. B., \& Jellison, W. L. (1933). Rocky Mountain spotted fever in Panama: report of two cases. The American Journal of Tropical Medicine and Hygiene, 1(4), 341-379. http://dx.doi.org/10.4269/ajtmh.1933. s1-13.341. PMid:14943913.

Paterson, S. (2015). Flea allergic dermatitis. Companion Animal, 20(3), 168-172. http://dx.doi.org/10.12968/ coan.2015.20.3.168.

Pugh, R. E. (1987). Effects on the development of Dipylidium caninum and on the host reaction to this parasite in the adult flea (Ctenocephalides felis felis). Parasitology Research, 73(2), 171-177. http://dx.doi.org/10.1007/ BF00536475. PMid:3575292.

Regendanz, P., \& Muniz, J. O. (1936). Rhipicephalus sanguineus como transmissor da piroplasmose canina no Brasil. Memorias do Instituto Oswaldo Cruz, 31(1), 81-84. http://dx.doi.org/10.1590/S0074-02761936000100005.

Schreck, C. E., Posey, K., \& Smith, D. (1978). Durability of permethrin as a potential clothing treatment to protect against blood-feeding arthropods. Journal of Economic Entomology, 71(3), 397-400. http://dx.doi.org/10.1093/ jee/71.3.397. PMid:690316.

Sen, S. K. (1933). The Vector of Canine Piroplasmosis due to Piroplasma gibsoni. Indian Journal of Veterinary Science, 3, 4.

Simpson, R. M., Gaunt, S. D., Hair, J. A., Kocan, K. M., Henk, W. G., \& Casey, H. W. (1991). Evaluation of Rhipicephalus sanguineus as a potential biologic vector of Ehrlichia platys. American Journal of Veterinary Research, 52(9), 1537-1541. PMid:1952347.

Zhao, X., Salgado, V. L., Yeh, J. Z., \& Narahashi, T. (2003). Differential actions of fipronil and dieldrin insecticides on GABA-gated chloride channels in cockroach neurons. The Journal of Pharmacology and Experimental Therapeutics, 306(3), 914-924. http://dx.doi.org/10.1124/jpet.103.051839. PMid:12766256.

Zhao, X., Yeh, J. Z., Salgado, V. L., \& Narahashi, T. (2004). Fipronil is a potent open channel blocker of glutamateactivated chloride channels in cockroach neurons. The Journal of Pharmacology and Experimental Therapeutics, 310(1), 192-201. http://dx.doi.org/10.1124/jpet.104.065516. PMid:15014137.

Zheng, Y., Priest, B., Cully, D. F., \& Ludmerer, S. W. (2003). RdlDv, a novel GABA-gated chloride channel gene from the American dog tick Dermacentor variabilis. Insect Biochemistry and Molecular Biology, 33(6), 595-599. http://dx.doi.org/10.1016/S0965-1748(03)00038-9. PMid:12770577. 